\title{
Inhibition of the Wnt palmitoyltransferase porcupine suppresses cell growth and downregulates the Wnt/ $\beta$-catenin pathway in gastric cancer
}

\author{
MIN-LI MO ${ }^{1 *}$, MENG-RU LI $^{2 *}$, ZHAO CHEN ${ }^{1}$, XING-WEI LIU ${ }^{3}$, QING SHENG ${ }^{2}$ and HAI-MENG ZHOU ${ }^{1,4}$ \\ ${ }^{1}$ Beijing Key Laboratory of Protein Therapeutics, School of Life Sciences, Tsinghua University, \\ Beijing 100084; ${ }^{2}$ College of Life Sciences, Zhejiang Sci-Tech University, Hangzhou, Zhejiang 310018; \\ ${ }^{3}$ Beijing ACCB Biotech Ltd., Beijing 100094; ${ }^{4}$ Zhejiang Provincial Key Laboratory of Applied Enzymology, \\ Yangtze Delta Region Institute of Tsinghua University, Jiaxing, Zhejiang 314006, P.R. China
}

Received November 29, 2012; Accepted February 20, 2013

DOI: $10.3892 / \mathrm{ol} .2013 .1256$

\begin{abstract}
Similarly to the Wnt protein palmitoyltransferase, porcupine (PPN) is essential to the activation of the $\mathrm{Wnt} / \beta$-catenin signaling pathway. However, little is known about the role of PPN activity in human gastric cancer, one of the most common causes of cancer-related mortality. Real-time quantitative PCR was used to detect the expression levels of PPN in paired gastric cancer tissues. Cell proliferation, migration and invasion assays were performed following treatment using a newly developed small molecule PPN inhibitor (inhibitors of Wnt production, IWP-2) in the gastric cancer MKN28 cell line. Expression of downstream target genes and transcriptional activity of the $\mathrm{Wnt} / \beta$-catenin signaling pathway were examined following IWP-2 treatment in MKN28. We identified that PPN was overexpressed in human gastric cancer tissue samples and cell lines. Following treatment of the gastric cancer cell line MKN28 with IWP-2, we detected that IWP-2 decreased MKN28 cell proliferation, migration and invasion, and elevated caspase 3/7 activity. Further analysis demonstrated that IWP-2 downregulated the transcriptional activity of the Wnt $/ \beta$-catenin signaling pathway and downregulated the expression levels of downstream Wnt/ $\beta$-catenin target genes in MKN28 cells. As current Wnt pathway-targeting strategies used for anticancer therapy have mainly focused on
\end{abstract}

Correspondence to: Dr Qing Sheng, College of Life Sciences, Zhejiang Sci-Tech University, No. 5 Second Avenue, Xiasha Higher Education Zone, Hangzhou, Zhejiang 310018, P.R. China

E-mail: csheng@zstu.edu.cn

Dr Hai-Meng Zhou, School of Life Sciences, Tsinghua University, No. 1 Qinghuayuan, Haidian, Beijing 100084, P.R. China

E-mail: zhm-dbs@tsinghua.edu.cn

${ }^{*}$ Contributed equally

Key words: Wnt, palmitoylation, palmitoyltransferase, porcupine, gastric cancer
Wnt-receiving cells, our data shed light on the potential use of Wnt palmitoyltransferase PPN inhibitors to abrogate Wnt production in Wnt-producing cells, thus providing a potential therapeutic option for gastric cancer.

\section{Introduction}

The Wnt signaling pathway is important in embryonic development as well as the initiation and progression of a number of types of human cancer (1-3). The Wnt/ $\beta$-catenin signaling pathway, also known as the canonical Wnt signaling pathway, involves the translocation of unphosphorylated $\beta$-catenin into the nucleus. In the nucleus, $\beta$-catenin interacts with transcription factors (including TCF or LEF) to initiate the transcription of downstream target genes, including C-MYC, CCND1 and BIRC5, to regulate cell proliferation, differentiation and survival (1).

As a Drosophila segment polarity gene, porcupine (PPN) encodes a transmembrane protein located in the endoplasmic reticulum which is necessary for the normal processing of Wingless protein (4). Although Hofmann predicted that PPN belongs to a superfamily of membrane-bound $\mathrm{O}$-acyltransferases (5), the active form of Wnt proteins were not identified to be palmitoylated on a conserved cysteine until 2003 (6). Several studies suggest that PPN-dependent palmitoylation is required for the activity and distribution of Wnt proteins (6-9).

Given that PPN acts as an essential palmitoyltransferase during the post-translational modification of Wnt proteins, a diverse library of synthetic small molecules have been screened to identify those that target Wnt-mediated cellular responses (10). One class of small molecules that inhibited the activity of PPN were named the inhibitors of Wnt production (IWP) (10). The IWP compounds share an identical core chemical structure and target the palmitoyltransferase PPN $(10,11)$. As the majority of currently used Wnt pathway-targeting strategies have focused on Wnt-receiving cells $(12,13)$, the potential use of IWP may provide new insights into the abrogation of aberrant Wnt signaling pathway activity in Wnt-producing cells and cancer therapy. 
Gastric cancer is one of the most common causes of cancer-related mortality worldwide (14). In China, the incidence rate of gastric cancer ranked as the third highest amongst the most common cancers in 2005 (15). The initiation and progression of gastric cancer have been linked to the aberrantly activated $\mathrm{Wnt} / \beta$-catenin signaling pathway (16-18). However, little is known on the role of PPN in gastric cancer. In this study, we first examined the expression profile of PPN in paired human gastric cancer tissue samples. We then investigated the effects of IWP on the cell growth and activity of the Wnt/ $\beta$-catenin signaling pathway in the gastric cancer cell line MKN28.

\section{Materials and methods}

Tissue samples. Tissue samples of sixteen gastric cancer patients from local hospitals were collected following receipt of written informed consent and approval by the Tsinghua University School of Medicine Ethical Review Committee, Beijing, China. Cancerous and adjacent normal tissues of the same patient were obtained during resection and immediately snap-frozen in liquid nitrogen. Normal tissues were purchased from Biochain (Newark, CA, USA). Tissue samples were stored at $-80^{\circ} \mathrm{C}$ prior to analysis.

RNA extraction, reverse transcription and real-time PCR. Total RNA was isolated from tissue or culture cell samples using the RNeasy Plus kit (Qiagen Inc., Valencia, CA, USA). The concentration of RNA was examined by Nanodrop 1000 (Thermo Fisher Scientific, Wilmington, DE, USA). Reverse transcription and real-time PCR were performed as previously reported (19). The sequences of primers and probes used were: for PPN, forward: 5'-CATCCTCATCTACCTACTCAT-3', reverse: 5'-CGCATCTTGTGCCATGTC-3', probe: 5'-CGG TGTCTACCATGTGCATCTC-3'; for internal control (ACTB), forward: 5'-GATCATTGCTCCTCCTGAGC-3', reverse: 5'-ACTCCTGCTTGCTGATCCAC-3', probe: 5'-CTCGCT GTCCACCTTCCAGCAGAT-3'; for AXIN2, forward: 5'-ACA TAGGTTCTGGCTATGTCTT-3', reverse: 5'-GTCAGCGCA TCACTGGATAT-3', probe: 5'-CCACCAGCGCCAACGACA GTG-3'; for C-MYC, forward: 5'-CCACGTCTCCACACAT CAG-3', reverse: 5'-TTGGCAGCAGGATAGTCCTT-3', probe: 5'-AACTACGCAGCGCCTCCCTCCAC-3'; for CCND1, forward: 5'-CGTCCATGCGGAAGATCGT-3', reverse: 5'-TCC TCCTCGCACTTCTGTT-3', probe: 5'-CTCGCAGAC CTCCAGCATCCAG-3'; for BIRC5 (encoding Survivin), forward: 5'-TGGAGTCTGGGAAGGGTTGT-3', reverse: 5'-GCTCTAACCTGCCATTGGAAC-3', probe: 5'-TCACCC ATAGCCCAGAAGCCTCA-3'. The $2^{-\Delta \mathrm{Ct}}$ value demonstrates the relative PPN expression (relative to internal control) in cancer cell lines and normal tissues. The $2^{-\Delta \Delta \mathrm{Ct}}$ value demonstrates the fold change of the relative PPN expression (relative to internal control) in cancer tissues normalized to adjacent normal tissues $(20)$ and $2^{-\Delta \Delta C t}>1.5$ was regarded as overexpression.

Cell culture and Wnt palmitoyltransferase inhibitor. Human gastric cancer cell lines MKN28 were obtained from the China Center for Type Culture Collection (Wuhan, China). The cells were cultured in Dulbecco's modified Eagle's medium supple- mented with $10 \%$ fetal bovine serum. Cells were cultured at $37^{\circ} \mathrm{C}$ in a humid incubator with $5 \% \mathrm{CO}_{2}$. Wnt palmitoyltransferase inhibitor (IWP-2) was purchased from Sigma Aldrich (St. Louis, MO, USA) and $30 \mu \mathrm{M}$ was used to treat the cells.

Western blot analysis. The detailed western blot procedures were performed as previously reported (21). The primary antibodies included anti- $\beta$-actin (1:5,000; Sigma Aldrich), anti- $\beta$-catenin (1:2,000; BD Biosciences, San Jose, CA, USA), anti-Axin 2 (1:1,000; Cell Signaling Technology, Inc., Danvers, MA, USA), anti-Cyclin D1 (1:2,000; Cell Signaling Technology, Inc.), anti-c-myc (1:1,000; Cell Signaling Technology, Inc.), anti-Survivin (1:1,000; Cell Signaling Technology, Inc.). $\beta$-catenin was detected in cytosolic proteins. All other targets were detected in total proteins.

Cell proliferation assay. CellTiter 96 Aqueous Non-Radioactive Cell Proliferation Assay kit (Promega Corporation, Madison, WI, USA) was used as previously reported (21).

Colony formation and soft agar assays. The detailed procedures for colony formation and soft agar assays were performed as previously reported (21).

Luciferase assay. Dual-Glo Luciferase Assay System (Promega Corporation) was used as previously reported (21). The ratio between firefly luciferase activity and renilla luciferase activity (FL/RL) was calculated to examine the $\mathrm{TCF} / \mathrm{LEF}$ transcriptional activity.

Caspase-3/7 activity assay. Caspase-Glo 3/7 Assay (Promega Corporation) was used for measuring caspase-3/7 activity according to the manufacturer's instructions. Treated cells $\left(100 \mu \mathrm{l}\right.$ of $\left.5 \times 10^{3}\right)$ were incubated with an equal volume of the assay reagent at room temperature. After $1.5 \mathrm{~h}$, luminescence was measured to calculate caspase-3/7 activity.

Transwell migration and invasion assay. Haptotax is chambers ( $8-\mu \mathrm{m}$ pore size, Corning Costar, Cambridge, MA, USA) without or with $5 \mathrm{mg} / \mathrm{ml}$ matrigel (Sigma Aldrich) were used for migration and invasion assays, respectively. The experiments were repeated three times independently.

Statistical analysis. Experimental data were analyzed using GraphPad Prism 5.0 for Windows (GraphPad Software, Inc., La Jolla, CA, USA). The differences in the relative expression levels of genes between two unpaired and paired groups were analyzed by the Student's t-test and the Wilcoxon matched pairs test, respectively. For independently repeated experiments, mean values \pm SD (error bars) are represented in Figs. 1-4. For all statistical tests, a two-tailed $\mathrm{P}<0.05$ was considered to indicate a statistically significant result. $\mathrm{P}<0.05, \mathrm{P}<0.01$ and $\mathrm{P}<0.001$ are represented as $*, * *$ and $* * *$, respectively.

\section{Results}

Overexpression of PPN in gastric cancer tissue samples and MKN28 cell line. PPN was overexpressed in $62.5 \%(10 / 16)$ of gastric cancer tissue samples compared with adjacent normal tissue samples (paired test, $\mathrm{P}<0.05$; Fig. 1A). We also noted 
A

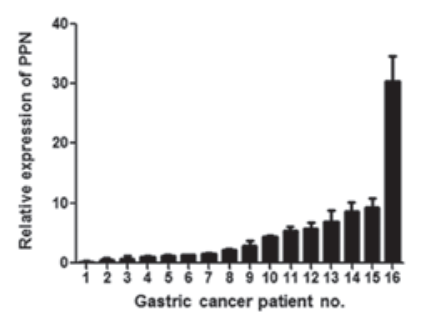

B

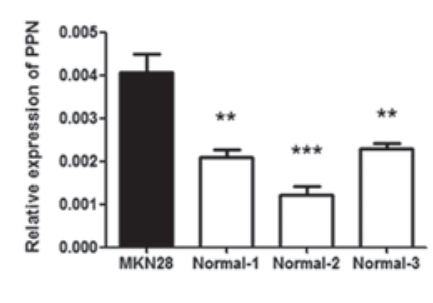

Figure 1. Overexpression of PPN in gastric cancer tissue samples and MKN28 cell line. (A) Real-time PCR analysis of PPN expression levels in paired gastric cancer tissue samples. The $2^{-\Delta \Delta C t}$ value demonstrates the fold change of the relative PPN expression (relative to internal control) in cancer tissue normalized to adjacent normal tissue. (B) Real-time PCR analysis of PPN expression levels in gastric cancer cell line MKN28 and three normal gastric tissue samples. The $2^{-\Delta C t}$ value demonstrates the relative PPN expression (relative to internal control). ${ }^{* *} \mathrm{P}<0.01,{ }^{*}{ }^{* *} \mathrm{P}<0.001$. PPN, porcupine

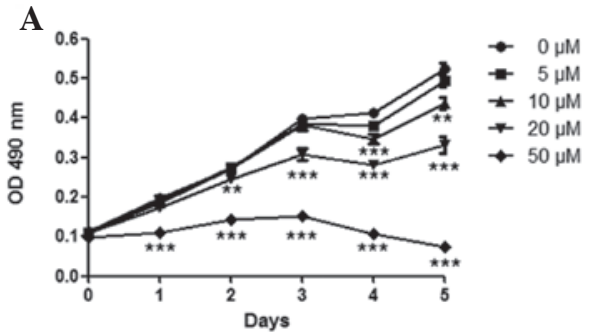

C

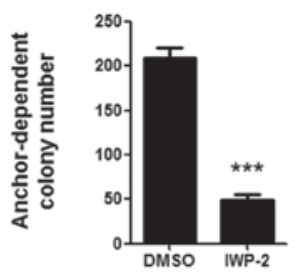

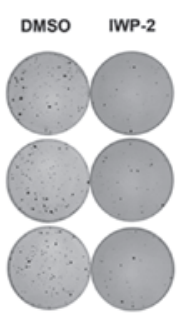

D

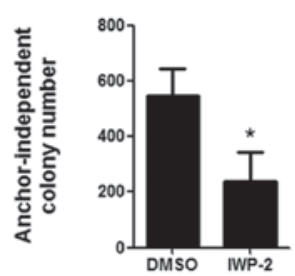

Figure 2. PPN inhibitor suppressed MKN28 cell growth. (A) The effect of IWP-2 at different concentrations $(0-50 \mu \mathrm{M})$ on MKN28 cell proliferation. Optical density, $490 \mathrm{~nm}$. (B) Anchor-dependent colony formation images. (C) Anchor-dependent colony number counts. The cells were treated with IWP-2 for 3 weeks. (D) Anchor-independent colony number counts. The cells were treated with IWP-2 for 3 weeks. ${ }^{*} \mathrm{P}<0.05,{ }^{* *} \mathrm{P}<0.01$ and ${ }^{* * *} \mathrm{P}<0.001$. PPN, porcupine; IWP, inhibitors of Wnt production.
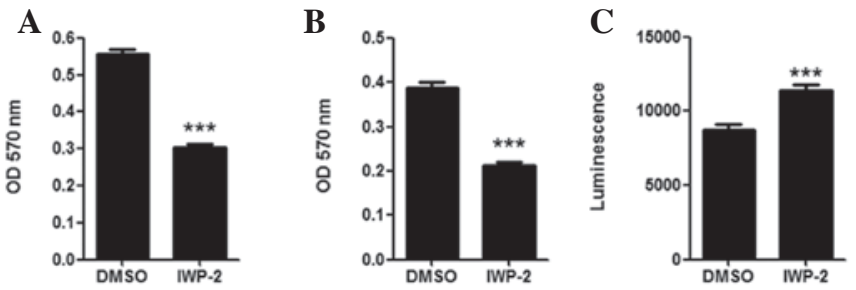

Figure 3. PPN inhibitor downregulated MKN28 cell migration and invasion, and induced cell apoptosis. (A) Transwell migration assay. (B) Invasion assay. (C) Caspase 3/7 activity assay. The cells were treated with IWP-2 for 3 days. Optical density, $570 \mathrm{~nm}$. ${ }^{* * *} \mathrm{P}<0.001$. PPN, porcupine.

that PPN expression in the gastric cancer cell line MKN28 was significantly higher than that in three normal gastric tissue samples that we examined $(\mathrm{P}<0.05$; Fig. 1B). These results indicate that PPN may be important in gastric cancer.
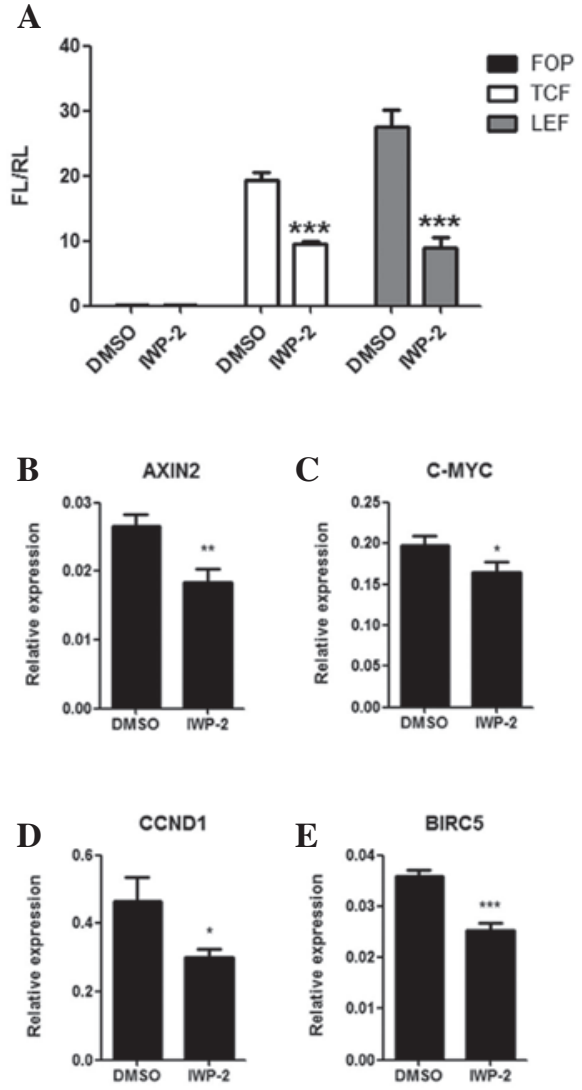

$\mathbf{F}$

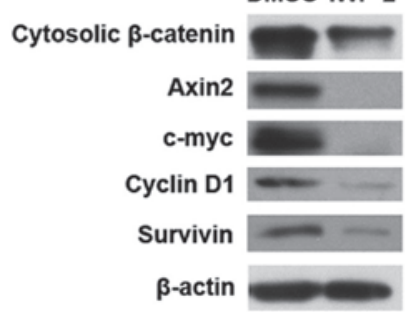

Figure 4. PPN inhibitor downregulated the activity of Wnt/ $\beta$-catenin signaling pathway. (A) Transcriptional activity of two transcription factors (TCF and LEF). FOP was used as the negative control. (B-E) Real-time PCR analysis of downstream $\mathrm{Wnt} / \beta$-catenin signaling pathway target genes (AXIN2, C-MYC, CCND1 and BIRC5, respectively). (F) Western blot for key regulators of the $\mathrm{Wnt} / \beta$-catenin signaling pathway. The cells were treated with IWP-2 for 3 days. ${ }^{*} \mathrm{P}<0.05,{ }^{* *} \mathrm{P}<0.01$ and ${ }^{* * *} \mathrm{P}<0.001$. PPN, porcupine; IWP, inhibitors of Wnt production.

PPN inhibitor suppresses MKN28 cell growth. To investigate the role of PPN as a Wnt palmitoyltransferase in gastric cancer, we used a palmitoyltransferase inhibitor (IWP-2) specific for PPN activity $(10,11)$. Following treatment in the MKN28 cell line for four days, 10-50 $\mu \mathrm{M}$ IWP-2 significantly suppressed the proliferation of MKN28 cells $(\mathrm{P}<0.05$; Fig. $2 \mathrm{~A})$. In addition, anchor-dependent and anchor-independent colony numbers were significantly decreased following IWP-2 treatment $(\mathrm{P}<0.05$; Fig. 2B-D).

PPN inhibitor downregulates MKN28 cell migration and invasion, and elevates caspase 3/7 activity. In cell migration and invasion assays, MKN28 cells demonstrated almost $50 \%$ reduction in migration (Fig. 3A) and invasion (Fig. 3B) 
following IWP-2 treatment $(\mathrm{P}<0.05)$. IWP-2 treatment also significantly elevated the cellular caspase $3 / 7$ activity in MKN28 cells $(\mathrm{P}<0.05)$, indicating that IWP-2 may induce cell apoptosis.

PPN inhibitor downregulates activity of the Wnt/ $\beta$-catenin signaling pathway. Due to the correlation of proliferation, migration and invasion of MKN28 cells with the activity of the $\mathrm{Wnt} / \beta$-catenin signaling pathway (21), we examined the effects of IWP-2 on the Wnt/ $\beta$-catenin signaling pathway in gastric cancer cells. We observed that the transcriptional activity of two transcription factors (TCF and LEF) were significantly decreased in MKN28 cells following IWP-2 treatment $(\mathrm{P}<0.05$; Fig. $4 \mathrm{~A})$. The mRNA and protein expression levels of four downstream Wnt $/ \beta$-catenin signaling pathway target genes (AXIN2, C-MYC, CCND1 and BIRC5) were also significantly downregulated in MKN28 cells following IWP-2 treatment ( $\mathrm{P}<0.05$; Fig. 3B-F). Furthermore, the expression levels of cytosolic $\beta$-catenin protein, a hallmark of Wnt $/ \beta$-catenin signaling pathway activation, was also decreased (Fig. 3F). Taken together, these data suggest that the inhibition of PPN abrogates activity of the Wnt/ $\beta$ catenin signaling pathway, providing more evidence of the importance of PPN activity in the aberrant activation of the Wnt/ $\beta$-catenin signaling pathway in gastric cancer.

\section{Discussion}

Although PPN has been demonstrated to be important in the processing and distribution of Wnt proteins (6-9), little is known with regard to its characteristics in human cancer. We previously identified that PPN was overexpressed in lung cancer (22). As gastric cancer is one of the most common causes of cancerrelated mortality worldwide and in China $(14,15)$, we aimed to study the role of PPN activity in gastric cancer. We identified that PPN was overexpressed in gastric cancer tissues and the MKN28 cell line. Notably, the Wnt/ $\beta$-catenin signaling pathway was aberrantly activated in gastric cancer tissues (18) and in the MKN28 cell line (21). Therefore, our results indicate that PPN overexpression correlates with the activation status of the $\mathrm{Wnt} / \beta$-catenin signaling pathway in gastric cancer.

We previously demonstrated that the proliferation of MKN28 cells correlated with the activity of the Wnt/ $\beta$-catenin signaling pathway in a Wnt ligand-dependent manner (21), suggesting the potential influence that PPN inhibition may exert on MKN28 cell proliferation. In the present study, we demonstrated that IWP-2, a newly developed small molecule PPN inhibitor (10), inhibited anchor-dependent and anchorindependent proliferation of MKN28 cells, providing evidence that inhibiting PPN activity may be a novel strategy for suppressing gastric cancer cell proliferation.

As downstream target genes of the $\mathrm{Wnt} / \beta$-catenin signaling pathway, CCND1 and C-MYC encode regulators of cancer cell proliferation, migration and invasion $(21,23,24)$. Therefore, downregulation of these genes following IWP-2 treatment correlated with downregulated activity of the Wnt/ $\beta$-catenin signaling pathway and inhibition of MKN28 cell proliferation, migration and invasion induced by IWP-2. In addition, downregulation of BIRC5, encoding Survivin which inhibits caspase activity and cell apoptosis (25), correlated with elevated activity of caspase 3/7 in MKN28 cells following IWP-2 treatment. These results provide further evidence for the use of PPN inhibitors to abrogate the aberrantly activated $\mathrm{Wnt} / \beta$-catenin signaling pathway.

In summary, we demonstrated that PPN expression levels are upregulated in gastric cancer tissues. We also revealed that IWP-2, a palmitoyltransferase inhibitor specific for PPN, inhibited cell proliferation, migration and invasion, as well as inducing apoptosis in gastric cancer cells. Further analysis showed that activity of the Wnt/ $\beta$-catenin signaling pathway was also downregulated by IWP-2 in these gastric cancer cells. Our data indicate that PPN activity may be critical for cell proliferation and activation of the Wnt/ $\beta$-catenin signaling pathway in gastric cancer. These data also provide evidence for the viability of targeting the Wnt/ $\beta$-catenin signaling pathway in Wnt-producing cells of gastric cancer and shed light on the potential use of PPN inhibitors as a therapeutic strategy for the treatment of gastric cancer.

\section{Acknowledgements}

This study was partially supported by the National Key Basic Research and Development (973) Program of China (No. 2011CB910800 and No. 2012CB917304), the China Natural Science Foundation (No. 31170732 and No. 31270854), the Natural Science Foundation of Zhejiang Province (No. Y204499) and a research fund from Beijing ACCB Biotech Ltd.

\section{References}

1. Polakis P: Wnt signaling and cancer. Genes Dev 14: 1837-1851, 2000.

2. Klaus A and Birchmeier W: Wnt signalling and its impact on development and cancer. Nat Rev Cancer 8: 387-398, 2008.

3. Clevers $\mathrm{H}$ and Nusse R: Wnt/ $\beta$-catenin signaling and disease. Cell 149: 1192-1205, 2012.

4. Kadowaki T, Wilder E, Klingensmith J, Zachary K and Perrimon N: The segment polarity gene porcupine encodes a putative multitransmembrane protein involved in Wingless processing. Genes Dev 10: 3116-3128, 1996.

5. Hofmann K: A superfamily of membrane-bound $\mathrm{O}$-acyltransferases with implications for wnt signaling. Trends Biochem Sci 25: 111-112, 2000.

6. Willert K, Brown JD, Danenberg E, et al: Wnt proteins are lipidmodified and can act as stem cell growth factors. Nature 423: 448-452, 2003.

7. Nusse R: Wnts and Hedgehogs: lipid-modified proteins and similarities in signaling mechanisms at the cell surface. Development 130: 5297-5305, 2003.

8. Galli LM, Barnes TL, Secrest SS, Kadowaki T and Burrus LW: Porcupine-mediated lipid-modification regulates the activity and distribution of Wnt proteins in the chick neural tube. Development 134: 3339-3348, 2007.

9. Coudreuse D and Korswagen HC: The making of Wnt: new insights into Wnt maturation, sorting and secretion. Development 134: 3-12, 2007.

10. Chen B, Dodge ME, Tang W, et al: Small molecule-mediated disruption of Wnt-dependent signaling in tissue regeneration and cancer. Nat Chem Biol 5: 100-107, 2009.

11. Dodge ME, Moon J, Tuladhar R, et al: Diverse chemical scaffolds support direct inhibition of the membrane-bound O-acyltransferase porcupine. J Biol Chem 287: 23246-23254, 2012.

12. Takahashi-Yanaga $\mathrm{F}$ and Kahn M: Targeting Wnt signaling: can we safely eradicate cancer stem cells? Clin Cancer Res 16: 3153-3162, 2010.

13. Chen W, Chen M and Barak LS: Development of small molecules targeting the Wnt pathway for the treatment of colon cancer: a high-throughput screening approach. Am J Physiol Gastrointest Liver Physiol 299: G293-G300, 2010. 
14. Jemal A, Bray F, Center MM, Ferlay J, Ward E and Forman D: Global cancer statistics. CA Cancer J Clin 61: 69-90, 2011.

15. Yang L: Incidence and mortality of gastric cancer in China. World J Gastroenterol 12: 17-20, 2006.

16. Du R, Xia L, Sun S, et al: URG11 promotes gastric cancer growth and invasion by activation of beta-catenin signalling pathway. J Cell Mol Med 14: 621-635, 2010.

17. Ganesan K, Ivanova T, Wu Y, et al: Inhibition of gastric cancer invasion and metastasis by PLA2G2A, a novel beta-catenin/TCF target gene. Cancer Res 68: 4277-4286, 2008.

18. Zhang $\mathrm{H}$ and Xue $\mathrm{Y}$ : Wnt pathway is involved in advanced gastric carcinoma. Hepatogastroenterology 55: 1126-1130, 2008.

19. Mo ML, Chen Z, Li J, et al: Use of serum circulating CCNB2 in cancer surveillance. Int J Biol Markers 25: 236-242, 2010.

20. Chen Z,Fan JQ, Li J, et al: Promoter hypermethylation correlates with the Hsulf-1 silencing in human breast and gastric cancer. Int J Cancer 124: 739-744, 2009.
21. Li J, Mo ML, Chen Z, et al: HSulf-1 inhibits cell proliferation and invasion in human gastric cancer. Cancer Sci 102: 1815-1821, 2011.

22. Chen Z, Li J, Li QS, et al: Suppression of PPN/MG61 attenuates Wnt/beta-catenin signaling pathway and induces apoptosis in human lung cancer. Oncogene 27: 3483-3488, 2008.

23. Zhang L, Hou Y, Ashktorab H, et al: The impact of C-MYC gene expression on gastric cancer cell. Mol Cell Biochem 344: 125-135, 2010.

24. Wolfer A and Ramaswamy S: MYC and metastasis. Cancer Res 71: 2034-2037, 2011.

25. Tamm I, Wang Y, Sausville E, et al: IAP-family protein survivin inhibits caspase activity and apoptosis induced by Fas (CD95), Bax, caspases, and anticancer drugs. Cancer Res 58: 5315-5320, 1998. 